V.v.H. and N.D.H. are International Scholars of the Howard Hughes Medical Institute.

Kiyoshi Miyagawa 1,6, Jill Kent', Adrian Moore', Jean-Paul Charlieu', Melissa H. Little', Kathleen A. Williamson', Anna Kelsey ${ }^{2}$, Keith W. Brown ${ }^{3}$, Shabbir Hassam4, Jakob Briner", Yasuhide Hayashi ${ }^{5}$, Hisamaru Hirai ${ }^{6}$, Yoshio Yazaki ${ }^{6}$, Veronica van Heyningen ${ }^{1}$ \& Nicholas D. Hastie ${ }^{1}$

'MRC Human Genetics Unit, Western General Hospital, Crewe Road, Edinburgh EH4 2XU,
UK. ${ }^{2}$ Department of Pathology, Royal Manchester Children's Hospital, Pendlebury, Manchester M27 1HA, UK. ${ }^{3}$ CLIC Research Unit, Department of Pathology and Microbiology, School of Medical Sciences, University Walk, Bristol BS8 ITD, UK. ${ }^{4}$ Departement Pathologie der Universitat, Institut fur Klinische Pathologie, Universitätsspital Zürich, Schmelzbergstrasse 12, CH-8091 Zurich, Switzerland. ${ }^{5}$ Department of Paediatrics and ${ }^{6}$ Third Department of Internal Medicine, University of Tokyo, 7-3-1 Hongo, Bunkyo-ku, Tokyo 113, Japan. Correspondence should be addressed to N.D.H

e-mail: oldnick@hgu.mrc.ac.uk

1. Hastie, N.D. Annu. Rev. Genet. 28, 523-558 (1994).

2. Call, K.M. et al. Cell 60, 509-520 (1990).

2. Call, K.M. et al. Cell 60, 509-520 (1990).
3. Gessler, M. et al. Nature 343, 774-778 (1990),

4. Drummond, 1.A. et al. Science 257, 674-678 (1992).

5. Larsson, S.H. et al. Cell 81, 392-401 (1995)

6. Weintraub, H. Cell 75, 1241-1244 (1993).

7. Olson, E.N. \& Klein, W.H. Genes Dev. 8, 1-8 (1994).

8. Little, M.H. et al. Hum. Mol. Genet. 2, 259-264 (1993).

9. Brown, K.W. et al. Oncogene 7, 763-768 (1992).

10. Bickmore, W.A. et al. Science 257, 235-237 (1992).

\title{
No female embryonic lethality in mice nullizygous for Msh2 and p53
}

$\mathrm{R}$ ecently, it has been reported that male mice bearing targeted inactivations of both $p 53$ and Msh 2 are viable, but rapidly succumb to lymphoma earlier than either $p 53$ or $M s h 2$ single mutants. In contrast, it was reported that all female mice lacking $p 53$ and Msh 2 underwent developmental arrest at 9.5 days and died in utero ${ }^{1}$. The published study did not report a significant reduction in the number of female $p 53^{-1-}$ animals, although the authors state that this is probably due to their small cohort size. We have also generated male mice lacking $p 53$ and Msh2 which are viable and succumb to lymphoma at a similarly early age ( $t^{1} / 2$ is 65 days). However, our results differ from the data of Cranston $e t$ al. in that we have successfully generated 22 adult female mice nullizygous for both $p 53$ and Msh2. The parental strains used to generate our cohort differ from those used by Cranston et al., but both have been well characterized previously ${ }^{2,3}$. Importantly, the single mutant $M s h 2$ and $p 53$ parental strains used in our cohort possess very similar reported phenotypes to those used by Cranston et al. ${ }^{2-5}$. Data from our cohort show the male to female ratio in $\mathrm{Msh} 2^{-/}$ $p 53^{-/-}$mice is similar to the ratio observed in $M s h 2^{+/+} p 53^{-1-}$ mice (Table 1). Thus, in our cohort $M \operatorname{sh} 2$ plays no detectable role in female development with the reduction in female mice in our $\mathrm{Msh} 2^{-/} p 53^{-/-}$cohort from expected Mendelian ratios being accounted for by $p 53$-associated exencephaly and subsequent anencephaly ${ }^{6}$. We observed an identical phenotype for female $\mathrm{Msh} 2^{-1-} \mathrm{p} 53^{-1-}$ mice as for their male counterparts, dying from lymphoma at a similar age. We have used male $M s h 2^{-1-}$ $p 53^{-1-}$ mice in successful breeding pairs, confirming that these mice are fertile.

\begin{tabular}{|c|c|c|}
\hline & $p 53^{+/+}$ & $p 53^{-1-}$ \\
\hline 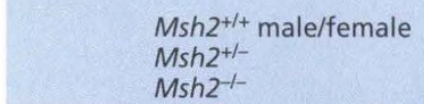 & $\begin{array}{l}262 / 254(-2 \%) \\
118 / 94(-11 \%) \\
166 / 177(+3 \%)\end{array}$ & $\begin{array}{l}26 / 12(-37 \%) \\
52 / 21(-42 \%) \\
61 / 22(-47 \%)\end{array}$ \\
\hline \multicolumn{3}{|c|}{$\begin{array}{l}\text { Sex ratio of mice for each genotype group together with the percentage deviation from the expected } 1: 1 \\
\text { male to female ratio shown in brackets. The observed reduction in female mice doubly null for Msh2 and } \\
\text { p53 is not significantly different from that related to } p 53 \text { deficiency alone ( } \chi 2 \text { test). In this data set } \\
\text { homozygosity for lack of } p 53 \text {, irrespective of Msh } 2 \text { status, confers a significant reduction in the number of } \\
\text { female progeny ( }<<0.01, \chi 2 \text { test). }\end{array}$} \\
\hline
\end{tabular}

We suggest four possible explanations for the difference between our own data and the work of Cranston et al. First, the discrepancy may result from different levels of environmental insult. However, our colony is not maintained under barrier conditions and we therefore consider this unlikely. Second, this difference may arise through the use of different genetic backgrounds. Indeed, we have previously documented the strain dependency of p53-related anencephaly $^{6}$. Against this possibility argues the fact that both groups of mice were generated from outbred crosses derived from a mixture of mouse strains (our animals segregate for 129/Ola, Balb-c and SWR genomes) and further, that our previous analysis showed different outcrossed strains to possess similar levels of p53-related embryonic death ${ }^{6}$. Notwithstanding these observations, it remains possible that the observed Msh2-related death is strain dependent, and we are currently carrying out appropriate backcrosses to address this point. Third, it is possible that the phenotype observed by Cranston et al. is arising as a consequence of a second mutation which is linked to one or other of the targeted alleles. The likelihood of such a linked mutation would perhaps be increased if either of these cohorts had been derived from ES cells engineered over an extended period in vitro to carry multiple mutations, but this was not the case $\mathrm{c}^{1-5}$. Finally, it is possible that the targetted events differ in some way, either at the targetted locus itself or by the influence of the targeted locus upon neighbouring genes. Although we cannot at present confirm or refute any of the above explanations, it is clear from our data that, at the very least, the reported female embryonic lethality associated with Msh2 and $p 53$ deficiency is not fully penetrant.

Acknowledgements

We thank H. te Riele for supply of mice. A.R.C. is a Royal Society University Research Fellow.

Neil J. Toft, Mark J. Arends,

Andrew H. Wyllie \& Alan R. Clarke Cancer Research Campaign Laboratories, Department of Pathology, University Medical School, Edinburgh, EH8 9AG, UK.

Correspondence should be addressed to A.R.C.

1. Cranston, A. et al. Nature Genet. 17, 114-118 (1997).

2. de Wind, N. et al. Cell 82, 321-330 (1995).

3. Clarke, A.R. et al. Nature 362, 849-852 (1993).

4. Donehower LA et al. Nature 356, 215-221(1992).

5 Reitmair, A.H. et al. Nature Genet. 11, 64-69 (1995).

6. Armstrong, J.F., Kaufman, M.H., Harrison, D.J. \& Clarke A.R. Curr. Biol. 5, 931-936 (1996). 